\title{
Les ressentis émotionnels, une entrée pour éduquer à l'égalité des sexes et à la littérature à l'école primaire
}

\section{Cendrine Marro, Gaël Pasquier et Laurence Breton}

\section{(2) OpenEdition \\ Journals}

Édition électronique

URL : http://journals.openedition.org/trema/3601

DOI : $10.4000 /$ trema.3601

ISSN : 2107-0997

Éditeur

Faculté d'Éducation de l'université de Montpellier

Édition imprimée

Date de publication : 1 décembre 2016

Pagination : 77-88

ISBN : 979-10-96627-02-8

ISSN : $1167-315 \mathrm{X}$

\section{Référence électronique}

Cendrine Marro, Gaël Pasquier et Laurence Breton, «Les ressentis émotionnels, une entrée pour éduquer à l'égalité des sexes et à la littérature à l'école primaire », Tréma [En ligne], 46 | 2016, mis en ligne le 01 avril 2017, consulté le 10 décembre 2020. URL : http://journals.openedition.org/trema/3601 ; DOI : https://doi.org/10.4000/trema.3601

Ce document a été généré automatiquement le 10 décembre 2020.

Trema 


\title{
Les ressentis émotionnels, une entrée pour éduquer à l'égalité des sexes et à la littérature à l'école primaire
}

\author{
Cendrine Marro, Gaël Pasquier et Laurence Breton
}

\section{Introduction}

1 Les professeur-e-s des écoles sont aujourd'hui de plus en plus incité-e-s à mettre en œuvre une éducation à la citoyenneté impliquant une grande diversité de thématiques dont celle de l'égalité des sexes (MEN, 2013; MEN, 2015). Au regard de cette thématique, une des approches classiques, depuis la publication de l'ouvrage d'Eléna Belotti, Du côté des petites filles (1973), est de dénoncer et de documenter le sexisme de la littérature jeunesse, y compris celle utilisée en classe, qu'elle réponde ou non aux recommandations ministérielles (Dafflon-Novelle, 2006; Brugeilles, Cromer et Panissal, 2009; Costes et Houadec, 2013). Dans la continuité de cette approche, un nombre croissant de publications ${ }^{1}$, et notamment de sites internet ${ }^{2}$, proposent aujourd'hui des fiches-analyses visant à sensibiliser aux stéréotypes et préjugés de sexe que véhiculent les ouvrages lus en classe. Toutefois, si aider les élèves à repérer ces éléments sexistes est important, les conduire à intégrer ces repères de manière à acquérir une sensibilité aux inégalités qui ne se limite pas à l'énonciation du principe d'égalité (qu'ils/elles sont généralement très prompt à exprimer) ne va pas de soi et nécessite un travail dans la durée. On peut dès lors s'interroger sur les gestes professionnels, notamment langagiers (Jorro, 2002), susceptibles de développer cette sensibilité ?

2 En vue de répondre à ce questionnement, une recherche action ${ }^{3}$ a été mise en place en septembre 2013. Elle s'inscrit dans le cadre conceptuel des études genre et plus spécifiquement dans celui des travaux de l'une d'entre nous sur la Dépendance- 
Indépendance à l'égard du Genre (DIG) qui précisément visent à formaliser et appréhender cette sensibilité aux inégalités de sexe (Marro, 2011 a et b, 2012). Programmée sur une durée de trois ans, son objectif est de penser l'éducation à l'égalité des sexes, non comme un objet éducatif supplémentaire déconnecté des enseignements habituels, mais intégrée à ceux-ci, par le biais d'une activité régulière centrale à l'école primaire : la lecture d'albums jeunesse ${ }^{4}$. Il ne s'agit pas toutefois d'utiliser la littérature comme un simple prétexte pour aborder cette thématique, pratique parfois privilégiée par certain-e-s enseignant-e-s du premier degré (Pasquier, 2013, 2015), mais bien de transmettre en classe, une culture de l'égalité des sexes tout en développant, chez les élèves, le goût pour la lecture active et critique. Dans cette perspective, et à condition de ne pas perdre de vue le travail sur la littérature, le recours au dispositif pédagogique du débat, encouragé par l'Éducation nationale ${ }^{5}$, notamment dans le cadre de l'Éducation Civique et Morale (EMC) nous a semblé un choix judicieux.

Bien que les enseignant-e-s impliqué-e-s dans le projet aient été très favorables à la mise en place de ces lectures-débats (qui, a priori, ne leur posaient pas problème), force nous a été de constater, au cours de la première année, combien la réalisation des débats rencontrait un certain nombre d'écueils récurrents (sur lesquels nous reviendrons plus loin), de l'école maternelle au CM2, entravant l'atteinte des objectifs fixés. Alors que cela ne figurait pas dans notre projet initial, ce constat nous a conduit-e-s à élaborer progressivement, durant la seconde année, un guide de questionnements visant à offrir un cadre support à ces lectures-débats. L'originalité de ce guide tient à ce qu'il se centre sur les ressentis émotionnels personnels des élèves afin de stimuler leur participation active et réflexive en lien avec la thématique travaillée. Bien que ce guide soit en cours d'expérimentation, dans une perspective pratique de partage d'expérience, il nous a semblé intéressant de pouvoir dès à présent en exposer les fondements ainsi que quelques réflexions et pratiques adaptatives qu'il a d'ores et déjà pu susciter. Avant d'en venir à sa présentation détaillée (partie III), nous exposerons les principaux aspects méthodologiques de la recherche action (partie I) puis, de manière synthétique et illustrée, les quelques écueils évoqués ci-dessus qu'il vise à dépasser (partie II).

\section{Méthodologie}

4 Sur la base du volontariat, une vingtaine d'enseignant-e-s (19 femmes, 1 homme) du département des Hauts-de-Seine, exerçant en école maternelle et élémentaire, de la grande section de maternelle au $\mathrm{CM} 2$, ont participé aux deux premières années de la recherche action support du présent article, soit huit en 2013/14 et treize en 2014/15 (12F-1H). Leur ancienneté dans la profession est très diverse. L'objectif étant d'accompagner ces enseignant-e-s dans la mise en œuvre d'une éducation à l'égalité des sexes par la littérature de jeunesse, le dispositif alterne des temps d'observation de lectures-débats en classe ${ }^{6}$ (observation directe ou indirecte via des enregistrements audio) et des temps de formation que nous avons animés (deux à trois dans une année scolaire donnée) $)^{7}$. Chaque classe doit réaliser au moins huit lectures d'albums dans l'année, organisant « une lecture en réseau » (Tauveron, 2002) sur le thème de l'égalité des filles et des garçons ${ }^{8}$.

5 Les pratiques de lectures-débats observées font l'objet d'échanges et de discussions après la séance avec les enseignant-e-s mais aussi durant les temps de formation afin de les améliorer et d'identifier les interventions propres à stimuler le débat (Pasquier, Marro et 
Breton, 2016). Le premier temps de formation, effectué en début d'année, consiste, outre à présenter le dispositif de la recherche action, à exposer brièvement notre cadre conceptuel de travail : celui des études genre et de la dépendance/indépendance à l'égard du genre (DIG). Dans ce contexte, les enseignant-e-s sont invité-e-s à opérationnaliser l'éducation à l'égalité des sexes par un accompagnement des élèves à repérer les inégalités/injustices (ce dernier terme étant souvent plus compréhensible pour les enfants), dont sont porteuses les différences de sexe qui jalonnent les albums jeunesse. Il s'agit ainsi de sensibiliser les enfants aux inégalités de sexe en les aidant à déshabiller les inégalités des habits de LA différence, soit des savoirs de sens commun concernant ce en quoi les filles et les garçons sont censés se distinguer, voire s'opposer, le plus souvent naturellement (Marro, 2012). Les temps de formation suivants sont davantage centrés sur les échanges d'expériences. Les données dont il sera question ici ont ainsi été obtenues lors des temps d'observation en classe, d'une part, et des temps de formation d'autre part.

Précisons qu'il ne s'agit nullement ici de prétendre à l'exhaustivité et encore moins à la représentativité des propos relatés et commentés. Ceux-ci n'ont d'autre objectif que de mettre l'accent sur des exemples, que nous jugeons significatifs, des questionnements professionnels que suscite le travail sur la littérature de jeunesse, articulé à une question socialement vive qui met en tension transmission de savoirs disciplinaires, éducation aux valeurs et mise en débat des savoirs de sens commun.

\section{De quelques difficultés dans la conduite des débats}

7 Un des premiers constats réalisés, dès la première ou seconde séance de classe, est que le dispositif de lecture débat sur le thème engagé, questionne l'ordinaire de l'utilisation de la littérature en classe pour en révéler les insuffisances, et ce, de la maternelle au CM2. Ainsi, en de nombreux points, nos observations font écho à ce que soulignent des rapports institutionnels ou des travaux antérieurs concernant le peu d'exploitations des albums dans un but pédagogique de sensibilisation à ou d'étude de la littérature. Par exemple, l'Inspection Générale de l'Éducation Nationale relève de manière sévère en 2011 dans son rapport consacré à l'école maternelle, qu'il y a «dans les classes beaucoup de lectures offertes mais peu de travail sur la compréhension : peu d'enseignants se soucient de la quantité et de la qualité du texte, de la nature des illustrations et de leur rapport avec le texte, de l'univers de référence, de la complexité du schéma de l'histoire et de sa structure, du nombre des personnages et de leurs comportements et/ou de leurs états mentaux, de la position du narrateur " (IGEN, 2011, p. 131). Dans le même ordre d'idée mais concernant cette fois l'école élémentaire, Catherine Tauveron expliquait en 1999 cette situation par l'aura que revêt la littérature aux yeux des enseignant-e-s. Cette position les amène à supposer que la simple mise en présence avec des histoires et des livres est suffisante pour établir une « » connivence culturelle et affective » entre le texte et l'élève » (pp. 9-10) et ainsi susciter le goût de lire comme la compréhension des textes. Certains propos relevés dans le cadre de notre recherche témoigne de la persistance d'une telle conception: «moi, je ne pose pas trop de questions d'habitude, on parle sur l'histoire naturellement en fait: j'arrête et ils parlent spontanément. Donc après, je recadre quand ils partent dans n'importe quel sens mais j'ai pas forcément de questions parce que les questions viennent un petit peu d'eux et de moi par rapport à ce qu'on a envie de se dire " (Séverine, GS). De leur côté, Laurence Pasa et Claire Beges ont 
également montré en 2006 que "les albums semblent moins utilisés comme point de départ à une activité de lecture [ou sur la littérature] que comme appui littéraire pour une autre matière» par les enseignant-e-s de Cycle 2 (p. 89). Confrontés à nos observations, ces constats nous ont conduit-e-s à penser que ce qui faisait surtout défaut aux professeur-e-s des écoles était l'usage d'outils permettant de mettre en œuvre ce que recommandent les documents d'accompagnements des programmes (MEN, 2002 et 2008), à savoir des stratégies destinées à travailler la compréhension des textes.

8 La question de l'égalité des sexes qui est ici étroitement associée au travail sur la littérature incite par ailleurs les enseignant-e-s à s'interroger sur le type de questionnement à mettre à œuvre pour lancer le débat. À l'instar de ce qu'ils-elles font habituellement, certain-e-s pensent qu'ils-elles doivent laisser un maximum d'élèves exprimer seuls, librement, toutes leurs idées suite à la lecture de l'album sans intervenir sur cette production: «Je ne pensais pas que je pouvais rebondir sur les idées émises par les élèves » (Nicolas, CM2). Cette posture les amène en général à opter au départ pour des questions relativement ouvertes telle cette enseignante qui choisit avec ses collègues de commencer par « et alors? ».

9 Elle constate rapidement l'échec de cette entrée «tellement ouverte que les enfants répètent l'histoire, inlassablement" (Séverine, GS) sans engager le débat. D’autres, parfois les mêmes en réaction à leurs premiers essais, repèrent alors combien « il faut être plus précis dans la question pour que [les élèves] puissent aller au-delà et s'identifier et évoquer les situations des personnages par rapport à eux-mêmes.» (Séverine, GS). Toutefois, ils-elles peinent à trouver leurs marques dans ce nouveau cadre, où elles ont l'impression d'orienter excessivement les réponses des élèves : « on en arrive à poser des questions fermées » (Isabelle, GS); « on a ce problème d'induction » (Fabienne, GS); « on pose des questions très précises donc au final on oriente la discussion » (Séverine, GS).

10 Ce dernier constat pose deux questions d'ordres différents mais qui interrogent également la possibilité même de débattre sur l'égalité des sexes. En premier lieu, alors que l'école inscrit l'égalité parmi les valeurs qu'elle entend défendre, qui la fondent et qui sont supposées la légitimer, ce principe d'égalité peut-il néanmoins y faire l'objet de débat? Car ces enseignantes n'imaginent pas, à juste titre, qu'à l'issue d'un débat, il soit possible de défendre l'idée que l'inégalité des sexes doit être promue. En second lieu, se pose pour ces enseignant-e-s la question de savoir comment il est possible de débattre alors qu'ils-elles attendent une réponse précise de la part de leurs élèves: "dans une lecture autre, il y a des réflexions spontanées.

11 On peut rebondir sur la réflexion d'un enfant et ça peut aller dans n'importe quel sens. Tandis que là on va vers une conclusion et on doit arriver finalement à une conclusion égalité... » (Séverine GS). Cette interrogation est d'autant plus vive que certaines enseignant-e-s remarquent que les élèves saisissent généralement très vite ce qui est attendu d'eux-elles. Certain-e-s professeur-e-s des écoles se demandent ainsi comment mettre les enfants en réflexion. En effet, une fois le principe de l'égalité des sexes admis, au moins en apparence, une fois énoncé dans le débat la phrase attendue selon laquelle " les filles et les garçons sont égaux ", que faire ? "Faut-il en rester là ? Là, les enfants en viennent tout le temps à dire : les garçons c'est comme les filles, les garçons ont le droit de pleurer, les garçons ont le droit de jouer avec des perles. Pour eux, c'est une évidence [...] C'est comme si on était un peu bloqués » (Laure, CM1); «j'ai exactement le même problème, surtout avec les CE2. Effectivement ils veulent faire consensus et puis ils sortent tous ça ; et puis arrivé là, on n'arrive pas vraiment à aller beaucoup plus loin » 
(Lucie, CE2 et CM2). Pour ces enseignant-e-s, cette simple affirmation du principe d'égalité par les élèves semble épuiser toute possibilité de questionnement. Pour nous, ces difficultés nous incitent à souligner l'importance de la distinction entre travailler à l'égalité des sexes par le débat et débattre de l'égalité des sexes. Or, c'est la première perspective qu'il nous intéresse d'opérationnaliser.

\section{Des constats à la proposition d'un guide de questionnements pour le débat}

12 Partant de ces observations et de nos échanges avec l'équipe, afin de formaliser l'accompagnement possible des élèves lors de la mise en œuvre des débats, nous avons opté pour l'élaboration d'un guide de questionnements. Soulignons que celui-ci n'a pas vocation à contraindre le déroulement ni même le contenu du débat, qui sont laissés à l'initiative des enseignant-e-s ayant reçu des éléments de formation pour ce faire. En effet, nous avons conscience que chaque album est une œuvre unique qui porte en ellemême son propre questionnement, et qu'il peut être nécessaire d'opter pour des formes d'interrogations différentes selon les niveaux de classe. Pour autant, afin de favoriser le travail collaboratif entre enseignant-e-s, nous avons été soucieux-ses de nous doter d'une méthodologie transmissible et transférable non seulement d'un album à l'autre mais également d'une classe à l'autre, de la maternelle au $\mathrm{CM}^{2}{ }^{9}$. En conséquence, notre proposition implique une série de huit questionnements qui peuvent ou non être déployés dans leur totalité, en respectant ou non l'ordre d'énonciation du guide voire être modifiés ou complétés par d'autres.

13 Ces questionnements s'inscrivent dans la continuité des écueils précédemment énoncés. Ils visent ainsi, tout en se dotant d'objectifs explicites quant au souci d'articuler travail en faveur de l'égalité des sexes et celui sur la littérature, à éviter d'une part que le débat ne parte dans tous les sens (et notamment ne se noie dans les savoirs de sens communs concernant LA différence des sexes) au risque de perdre de vue la question de l'égalité ; à faire en sorte d'autre part qu'il ne s'enlise pas rapidement dans l'énoncé superficiel du principe d'égalité des sexes. Pour répondre à ces objectifs, il nous a paru intéressant de recourir à un élément qui, à de rares exceptions, n'était pas sollicité dans les séances que nous avons observées, mais que les recherches en psychologie sociale sur les préjugés tout comme celles en didactique de la littérature mobilisent: la dimension affectivoémotionnelle. Les premières suggèrent en effet qu'être capable de se mettre à la place d'un membre d'un groupe discriminé et de saisir les émotions qu'il ressent (empathie) augmente la perception d'injustice et améliore l'attitude envers cette personne. Cette attitude positive sera alors généralisée au groupe d'assignation de la personne habituellement discriminée (Legal et Delouvee, 2008). Favoriser cette empathie est ainsi un des ressorts classiques pour lutter contre les préjugés.

Les secondes considèrent quant à elles que comprendre affectivement les personnages est un moyen fondamental de comprendre l'histoire et d'anticiper son déroulement (Giasson, 2005-b) au point qu'il s'agirait d'une compétence distinctive des lecteur-rice-s expert-e-s (Barton, 1996). D'autres travaux suggèrent par ailleurs que « la sollicitation du registre des émotions " (Croce-Spinelli, 2006, p.58) constitue une forme de questionnement féconde à la fois dans le cadre des débats interprétatifs et pour susciter le goût de lire (Merlin-Kajman, 2016). 
Nous avons ainsi choisi d'élaborer notre guide sur cette dimension affectivo-émotionnelle pour questionner la réception des œuvres au regard de notre thématique, en nous centrant sur huit émotions: les six émotions de base classiquement distinguées en psychologie, soit la joie, la tristesse, la peur, la colère, la surprise, le dégoût ; ainsi que deux autres émotions plus complexes, la honte et la fierté. Ces deux dernières émotions nous apparaissent intéressantes à solliciter dans le cadre d'un travail réflexif empathique visant à l'identification des inégalités/injustices dont sont porteuses différentes situations. En effet, ce n'est pas tant d'égalité de droit au sens formel du terme dont il est question ici, mais bien de favoriser l'égalité réelle, en développant la sensibilité aux inégalités. Celle-ci doit permettre de construire le sentiment d'égalité subjective, soit le sentiment d'être l'égale de l'autre, afin justement de réussir à se conduire et construire comme tel-le.

Huit grandes catégories de questions interpellant cette dimension de manière plus ou moins directe ont été retenues (cf. tableau ci-dessous) : quatre concernent le ressenti émotionnel de l'élève (Q1-Q2-Q3) et des personnages (Q5); deux incitent à une mise en lien histoire/personnages avec des expériences potentiellement vécues hors puis dans l'école (Q4 et Q7); les deux catégories de questions restantes portent plus directement sur les relations/rapports entre filles et garçons manifestées dans l'histoire et leurs conséquences inégalitaires/injustes (Q6 et Q8).

L'ordre d'apparition de ces questionnements dans le guide répond à une logique d'expression allant du ressenti le plus général à l'interprétation de la situation en termes d'inégalité/injustice. Notre souci d'ancrer le débat en partant du ressenti émotionnel fait que nous conseillons d'énoncer la première question avant même la lecture de l'album afin de bien mobiliser l'attention des élèves sur cette dimension durant cette lecture. Reprise immédiatement à la fin de l'histoire, elle permet de donner un fil conducteur aux premiers échanges, sans les enfermer d'emblée dans la thématique de l'égalité fillesgarçons.

18 Pour introduire le débat, les enfants sont donc invités et aidés à exprimer leur ressenti émotionnel (Question 1), puis à associer à ce ressenti une prise de position personnelle concernant ce qui leur a particulièrement plu ou déplu dans l'histoire (moment ou personnage-Question 2) ou encore un ressenti interrogatif renvoyant à ce qui les a étonnés, ce qui leur pose question (Question 3) pour enfin évoquer éventuellement un ressenti expérientiel personnalisé général, non cantonné à leur expérience scolaire mais renvoyant au souvenir de quelque chose de vécu, vu ou entendu ravivé par l'histoire (Question 4).

19 Vient ensuite une série de questions permettant de creuser plus spécifiquement la proximité affective des élèves avec les personnages (Question 5) en lien avec les stéréotypes, les normes ou les inégalités dont ceux-ci sont porteurs ou pas. L'objectif est ici de susciter leur empathie afin qu'ils-elles saisissent explicitement les répercussions émotionnelles de comportements ou attitudes porteuses d'injustices ou d'inégalités. L'ensemble des questionnements prévus peut varier quelque peu suivant l'histoire, notamment en les orientant davantage sur certains propos tenus ou comportements adoptés par les personnages que ceux-ci revendiquent le respect des normes de sexe ou s'y opposent, et les raisons selon elles et eux de ces propos, de ces comportements. Chaque étape du guide peut nécessiter pour l'élève de se référer au texte ou aux images de l'album pour justifier son propos, y chercher des informations dont il-elle a besoin. Toutefois, cette question 5 s'inscrit plus particulièrement dans un travail de 
compréhension et d'interprétation littéraire classique, centré sur la notion de personnage ou la structure du récit... (Tauveron, 2002; Giasson, 2005 a et b, 2008), tel que le connaissent bien souvent les professeur-e-s des écoles même s'ils-elles n'y recourent qu'à des degrés divers en classe (Tauveron, 1999; Pasa et Beges, 2006; IGEN 2011). Notre objectif est que les enseignant-e-s puissent articuler cette approche avec des préoccupations liées à l'éducation à l'égalité des sexes. S'insère à notre sens très bien dans cette série, un questionnement permettant d'accéder de manière moins directe mais tout aussi signifiante au ressenti émotionnel et qui invite chacun-e à indiquer si elle-il aurait envie ou pas de faire de tel ou tel personnage, un copain ou une copine. D'une certaine manière, elle vise également à articuler lecture distanciée et lecture fusionnelle au sens où l'entend Catherine Tauveron (2002).

Ce dernier questionnement est à notre sens propice à introduire, par le biais des justifications énoncées, la 6e catégorie de questions: Perceptions des modalités de relations sexuées (Question 6), qui elle-même permet de cheminer vers la perception des inégalités/injustices en lien avec la stimulation de la lecture en réseau (Question 8) en passant par le ressenti expérientiel contextualisé à l'école (Question 7). Ces questions invitent les enseignant-e-s à préciser avec leurs élèves ce qu'ils-elles entendent par égalité et inégalité afin d'affiner de manière adaptée à l'âge des enfants, selon les contextes et au fur et à mesure des rencontres littéraires, le contenu de ces notions et leur complexité. On sera particulièrement attentif/tive ici à aider les enfants à distinguer " être identique/pareil-le-s " et " être égaux/égale », termes entre lesquels bon nombre d'enfants, comme d'adultes, établissent des relations de cause à effet, voire qu'ils-elles utilisent comme synonymes. Dans la continuité du rappel des lectures précédentes et donc de la mise en réseau, il s'agit d'accompagner les enfants à repérer de manière de plus en plus autonome, les inégalités (injustices) que produisent les différences.

\section{Discussion conclusive}

21 Le guide que nous venons de présenter constitue une proposition pour outiller les enseignant-e-s. Il permet de mettre l'accent sur ce que peut concrètement signifier pour des professeur-e-s des écoles d'engager avec leurs élèves une réflexion sur l'égalité des sexes en lien avec la littérature de jeunesse. Son originalité est de chercher à atteindre cet objectif en se centrant sur les émotions. En cela, il rompt avec les gestes professionnels habituellement sollicités par les enseignant-e-s que nous avons observés, ce que confirment les réactions d'un certain nombre lors de sa présentation ou de sa première expérimentation.

En effet, des attentes de reformulations de l'histoire supposées attester de sa bonne compréhension guident bien souvent les premières interactions enseignant-e-s/élèves, qu'elles s'expriment ou non expressément. De fait, ce guide qui propose une entrée par le ressenti émotionnel a mis des enseignant-e-s mal à l'aise en occultant cette étape qui leur semble capitale. Dans la mesure où il leur était possible dans une perspective d'appropriation, de ne pas poser toutes les questions ou d'en intervertir l'ordre, leur stratégie a alors été de passer directement à la question 5 concernant les personnages, plus en phase avec des gestes professionnels qui leurs sont familiers. Quant à celles-ceux qui n'ont pas éprouvé ce malaise ou l'ont dépassé, ils-elles ont bien souvent pu constater qu'enferrés dans leurs habitudes, leurs élèves n'entendaient pas la question et 
reformulaient l'histoire, ou encore parlaient des émotions des personnages et non des leurs.

Ce constat nous a conduit à proposer la mise en œuvre d'une séance préalable sur les émotions afin d'aider à leur identification et à leur expression, en laissant chaque enseignant-e libre de l'organiser comme il-elle l'entend. Ainsi, peut être abordé un nombre plus ou moins important d'émotions, en les associant ou non à une représentation figurative comme des pictogrammes, qui peut être utilisée lors des lectures. Ce choix est susceptible de varier selon l'âge des élèves et la nouveauté que représente une telle entrée dans l'approche des livres dans la class $\mathrm{e}^{10}$. Ce travail préalable sur les émotions s'est avéré faciliter l'usage du guide, pour les enseignant-e-s comme pour les élèves. Il a par ailleurs pu être développé sur plusieurs séances en raison des nouvelles perspectives de réflexion et d'expression qu'il ouvrait et de l'intérêt des enfants.

En conclusion, de par les questionnements qui le composent, le guide n'incite pas les élèves à se positionner vis-à-vis du principe d'égalité filles-garçons dans lequel les débats avaient auparavant tendance à s'enliser assez rapidement.

En revanche, partant de l'identification de ressentis émotionnels différemment contextualisés, il les amène à davantage s'approprier l'album y compris dans sa dimension littéraire, et les aide à démasquer les inégalités dont sont porteuses des situations qui assignent filles et garçons à des rôles présentés comme simplement, voire naturellement, différents, et qui sont en fait porteurs d'inégalités.

Tableau 1 : Guide de questionnements proposés. Pour chaque question, les élèves sont incités à argumenter leurs réponses.

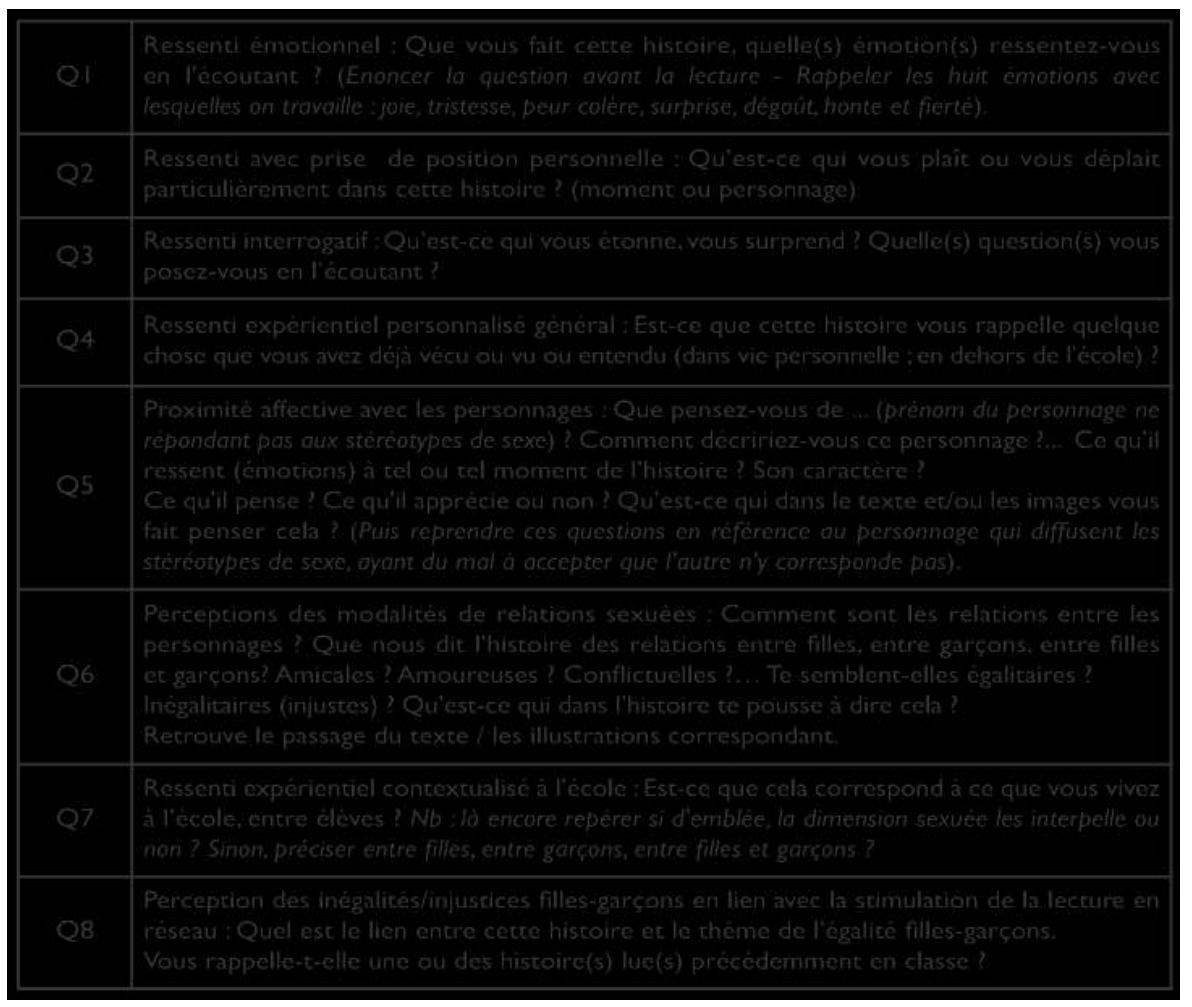




\section{BIBLIOGRAPHIE}

Barton, J. (1996). Interpreting character emotions for literature comprehension. Journal of Adolescent and Adult Literacy, 40 (1), 22-28.

Belotti, E. (1973). Du côté des petites filles. Paris : Éditions des femmes.

Breton, L., Marro, C., et Pasquier, G. (2016 à paraître). Le carnet de littérature, un outil pour réfléchir en classe à l'égalité des sexes ? In C. Mongenot et $\mathrm{S}$. Ahr (Dir.), (D)écrire, prescrire, interdire : les professionnels face à la LJ aujourd'hui. Paris : EMA/AGORA/CANOPE.

Brugeilles, C., Cromer, S. et Panissal, N. (2009). Le sexisme au programme ? Représentation sexuées dans les lectures de référence à l'école. Travail, genre et société, 21 (1), 107-129.

Costes, J. et Houadec, V. (2013). La construction du genre à travers les images des couvertures de littérature de jeunesse. In C. Morin-Messabel (Dir.), Filles/Garçons. Questions de genre, de la formation à l'enseignement (pp. 465-492). Lyon : Presses Universitaires de Lyon.

Croce-Spinelli, H. (2006). Lecture littéraire des élèves et variations des gestes de l'enseignant ». Les Dossiers des sciences de l'éducation, 15, 53-61.

Dafflon Novelle, A. (2006). Littérature enfantine : entre images et sexisme. In A. Dafflon Novelle (Dir.), Filles-garçons. Socialisation différenciée? (pp. 303-324). Grenoble : Presses Universitaires de Grenoble.

Ferrière, S. et Morin-Messabel, C. (2014). Contre-stéréotypes et développement de l'identité de genre. Impact des lectures d'albums en maternelle. In C. Connan-Pintado et G. Behoteguy (Dir.), Etre une fille, un garçon dans la littérature pour la jeunesse. France 1945-2012 (pp. 225-238). Bordeaux : Presses Universitaires de Bordeaux.

Giasson, J. (2008). La compréhension en lecture. Bruxelles : De Boeck.

Giasson, J. (2005-a). La lecture. De la théorie à la pratique. Bruxelles : De Boeck.

Giasson, J. (2000-b). Les textes littéraires à l'école, Bruxelles : De Boeck.

Inspection Générale de l'Éducation Nationale (2011). L'école maternelle. Paris : Ministère de l'Éducation nationale.

Jorro, A. (2002). Professionnaliser le métier d'enseignant. Paris : ESF.

Legal, J.-B. et Delouvée, S. (2008). Stéréotypes, préjugés et discrimination. Paris : Dunod.

Lesaux, E. et Marro, C. (2015). D'une analyse collaborative des stéréotypes de sexes et divisions sexuées dans la littérature pour la jeunesse, présente dans les classes, à l'implication des enseignant-e-s, participant à une recherche-action, dans l'éducation à l'égalité des sexes. Communication au colloque international thématique de l'AECSE, « Le Genre dans les sphères de l'éducation, de la formation et du travail ; Mises en images et représentations ", Reims, 28-30 octobre 2015.

Marro, C. (2012). Dépendance-indépendance à l'égard du genre. Penser l'égalité des sexes au-delà de LA différence. Recherche et Formation, 69, 65-80.

Ministère de l'Éducation nationale (MEN) (2013). Convention interministérielle pour l'égalité entre les filles et les garçons, les femmes et les hommes dans le système éducatif 2013-2018. Paris : Éducation nationale. 
Ministère de l'Éducation nationale (MEN) (2013). Programme d'enseignement moral et civique. Bulletin Officiel de l'Éducation nationale spécial, 6, 25 juin 2015.

Morin-Messabel, C. et Ferrière, S. (2013). Albums contre-stéréotypés et lecture offerte en Grande Section de Maternelle : mesure de l'impact sur les élèves à travers le dessin et la dictée à l'adulte. In C. Morin-Messabel et M. Salle (Dir.). À l'école des stéréotypes. Comprendre et déconstruire (p. 43-74). Paris : L'Harmattan.

Merlin-Kajman, H. (2016). Lire dans la gueule du loup. Essai sur une zone à défendre, la littérature. Paris : Gallimard.

Ministère de l'Éducation nationale (2002/2008). Une culture littéraire à l'école. Paris : Scéren-CNDP. Document disponible en ligne $:$ http://media.eduscol.education.fr/file/ecole/46/9/culturelitteraire-ecole_121469.pdf [Consultée le 17/11/2015]

Pasquier, G. (2013). Les pratiques enseignantes en faveur de l'égalité des sexes et des sexualités à l'école primaire, vers un nouvel élément du curriculum. Thèse de doctorat, Université Paris Ouest Nanterre La Défense.

Pasquier, G. (2015). Égalité des sexes à l'école : prévenir les malentendus scolaires. Carnets rouges, 2, 30-33. Article disponible en ligne, http://reseau-ecole.pcf.fr/sites/default/files/

cr_numero2.pdf [Consultée le 17/11/2015]

Pasquier, G., Marro, C. et Breton, L. (2016 à paraître). Éduquer à l'égalité des sexes à l'école primaire : autour de quelques gestes professionnels accompagnant une activité de lecture littéraire. Le Français aujourd'hui, 193.

Tauveron, C. (1999). Comprendre et interpréter la littérature à l'école : du texte réticent au texte proliférant. Repères, 19, 9-38.

Tauveron, C. (Dir.). Lire la littérature à l'école Pourquoi et comment conduire cet apprentissage spécifique? De la GS au CM. Paris : Hatier.

\section{NOTES}

1. À titre d'exemples: Textes et Documents pour la Classe (2001). Filles et garçons dans la littérature de jeunesse. tdc, 823 ; Houadec, V. et Babillot, M. (2008). 50 activités pour l'égalité entre filles et garçons à l'école. Toulouse : SCEREN- CRDP Midi-Pyrenées ; Chabrol Gagne, N. (2011). Filles d'albums. Les représentations du féminin dans l'album. Le Puy-en-Velay : L'atelier du poisson soluble; SNUIPP (2013/2015). Éduquer contre l'homophobie dès l'école primaire. Des outils théoriques et pratiques pour avancer. Document disponible en ligne http:// www.snuipp.fr/IMG/pdf/document_telechargeable-2013-30-05.pdf [Consultée le 29/04/2016].

2. À titre d'exemples les sites internet de l'association Adéquations, des éditions Talents Hauts, ou de Fille d'album. Il faudrait également ajouter les fiches pédagogiques concernant la littérature de jeunesse publiées par le ministère de l'Éducation nationale et le ministère des Droits des Femmes dans le cadre du programme ministériel des ABCD de l'égalité durant l'année scolaire 2013-2014 : Lagarde, F., Rouyer-Marie, F. et Javerzat, M.-C. (2013). Parcours : la figure de la belle (fiche 1 et 2). Elles ont cependant été retirées du site par ces ministères suite à l'abandon du programme.

3. Prenant appui sur un groupe d'enseignant-e-s des Hauts-de-Seine avec lequel l'une d'entre nous, Laurence Breton, en tant que conseillère pédagogique, expérimentait, depuis 2012/2013, des pratiques de classe autour de lecture-débats d'albums jeunesse afin de favoriser le 
développement de compétences sociales et civiques, en lien avec le respect de l'autre et de l'égalité filles-garçons.

4. D'autres recherches utilisent un tel support dans une perspective différente de la nôtre comme celle de Christine Morin-Messabel et Séverine Ferrière portant sur l'impact des lectures contrestéréotypées auprès des enfants de Grande Section (Morin-Messabel et Ferrière, 2013 ; Ferrière et Morin-Messabel, 2014). Une autre recherche en cours sur cette thématique, base de la réalisation d'une thèse en sciences de l'éducation réalisée par Elsa Lesaux, co-dirigée par Cendrine Marro et Marie-Anne Hugon montre que le comportement de chercheur-e-s en herbe adopté par les élèves stimulés par les enseignant-e-s permet de favoriser l'appropriation de la thématique par ces mêmes élèves tout en stimulant en retour l'implication des enseignant-e-s (Lesaux et Marro, 2015).

5. La pratique du débat facilite particulièrement la construction du jugement moral et du civisme chez les élèves. En ce sens, elle se situe au cœur d'une éducation à la citoyenneté. Cf. fiche Eduscol «Le débat (réglé ou argumenté)». http://cache.media.eduscol.education.fr/file/ EMC/01/1/ress_emc_debat_464011.pdf. [Consultée le 29/04/2016].

6. D'un durée d'une heure maximum, ces lectures débats sont suivies directement ou dans la semaine d'un travail d'écriture dans un carnet de littérature dont il ne sera pas question ici. Pour une description détaillée de la méthode, se reporter à Breton, Marro et Pasquier, 2015.

7. Cette formation s'intégrait dans le cadre de la formation continue des enseignant-e-s. Sa durée était donc contrainte et correspondait à $6 \mathrm{~h}(2 \times 3 \mathrm{~h})$ la première année et $9 \mathrm{~h}(3 \times 3 \mathrm{~h})$ la deuxième année, impliquant ainsi nécessairement une séance de mises au point conceptuelle et pratique en début d'année et une séance bilan en fin d'année.

8. Ces albums n'étaient pas nécessairement supposés traiter explicitement d'égalité des sexes. Cependant, ils devaient permettre de mettre en relief et en débat combien les différences filles/ garçons, censées distinguer toutes les filles de tous les garçons et réciproquement, pouvaient très concrètement, dans différents contextes se traduire en termes d'inégalités/injustices pour l'un ou l'autre sexe.

9. Ce choix nous a semblé d'autant plus important que certains enseignant-e-s d'une même école travaillant dans des classes de niveaux différents participaient au projet et que les professeur-e-s des écoles sont susceptibles de par leurs fonction d'enseigner dans tous les niveaux de classe, de la maternelle en élémentaire au cours de sa carrière.

10. Un tel travail peut par ailleurs être outillé par certains ouvrages existants destinés à la jeunesse : Browne, A. (2011). Parfois je me sens... Paris : Kaléidoscope ; Dumontet, A. et Langlois, A. (ill.) (2014). Les émotions. Paris : Milan; Herbera, G. (2010). Monsieur Cent Têtes. Paris : Mémo.

\section{RÉSUMÉS}

Cet article prend appui sur une série de constats réalisés dans le cadre d'une recherche-action conduite avec des enseignant-e-s de l'école primaire, recherche qui porte sur l'utilisation d'un dispositif de lectures-débats dans le but d'éduquer à l'égalité des sexes tout en développant le goût pour la lecture. Il explicite le contenu d'un guide de questionnements incitant à l'identification et à l'expression du ressenti émotionnel des élèves et des personnages, élaboré suite aux écueils récurrents constatés dans la réalisation des débats par les enseignant-e-s. 
This article is based on a series of findings made in the context of an action-research conducted with primary school teachers. This research deals with the use of a readings-and-debates tool which aims to educate to sex equality while developing the taste for reading. It explains the content of a questioning guide that promotes the identification and expression of the emotional feelings of the pupils and the characters. This guide was drawn up following recurring limits identified in the pursuit of discussions by teachers.

\section{INDEX}

Mots-clés : genre, littérature de jeunesse, pratiques enseignantes, égalité des sexes, ressenti émotionnel

Keywords : gender, children's literature, teaching practices, sex equality, emotional feeling

\section{AUTEURS}

\section{CENDRINE MARRO}

Maîtresse de conférences HDR, UFR des sciences psychologiques et sciences de l'éducation, Université Paris Ouest Nanterre La Défense, Laboratoire d'études de genre et de sexualité (LEGS) UMR 8238

\section{GAËL PASQUIER}

Maître de conférences en sociologie, ESPE - Université Paris Est Créteil (UPEC), Observatoire Universitaire International Éducation et Prévention (OUIEP), Laboratoire Interdisciplinaire de Recherche sur les Transformations des pratiques Éducatives et des pratiques Sociales (LIRTES)

\section{LAURENCE BRETON}

Conseillère pédagogique chargée de la Maîtrise de la langue et de l'Enseignement moral et civique, Université de Versailles 